\title{
Effect of Pressure on Statics, Dynamics, and Stability of Multielectron Bubbles
}

\author{
J. Tempere, ${ }^{1,2}$ Isaac F. Silvera, ${ }^{1}$ and J. T. Devreese ${ }^{2, *}$ \\ ${ }^{1}$ Department of Physics, Harvard University, 14-A Oxford Street, Cambridge, Massachusetts 02139 \\ ${ }^{2}$ Department of Natuurkunde, Universiteit Antwerpen, Universiteitsplein 1, B2610 Antwerpen, Belgium
}

(Received 24 August 2001; published 7 December 2001)

\begin{abstract}
The effect of positive and negative pressure on the modes of oscillation of a multielectron bubble in liquid helium is calculated. Already at low pressures of the order of 10-100 mbar, these effects are found to significantly modify the frequencies of oscillation of the bubble. Stabilization of the bubble is shown to occur in the presence of a small negative pressure, which expands the bubble radius. Above a threshold negative pressure, the bubble is unstable.
\end{abstract}

DOI: $10.1103 /$ PhysRevLett.87.275301

Multielectron bubbles (MEBs) in liquid helium are fascinating entities expected to display novel resonant behavior, the possibility of superconductivity, and sufficient electron density for Wigner crystallization and quantum melting. MEBs are bubbles inside the helium, containing only electrons that form a curved two-dimensional electron gas (2DEG) on the spherical surface of the bubble, the width of the thin spherical shell that conforms to the helium surface being of order 5-20 $\AA$ [1,2], compared to $70-80 \AA$ on a flat surface [3]. New efforts to trap and localize MEBs for long periods of time [4] have led to further consideration of the long term stability of bubbles. In this Letter we discuss the use of pressure and, in particular, negative pressure to stabilize MEBs against dynamic instabilities.

The radius of the bubble depends on the enclosed charge. In a simplified model, valid for large bubbles, the radius is given by $R_{C}=\left[e^{2} N^{2} /(16 \pi \sigma \varepsilon)\right]^{1 / 3}$, where $e$ is the electron charge, $N$ is the number of electrons in the bubble, $\sigma$ is the surface tension of helium, and $\varepsilon$ is the dielectric constant of helium [1]. A single electron bubble has a radius of $17.2 \AA$ [5] while, for example, a bubble of $10^{4}$ electrons has a theoretical radius of $1 \mu \mathrm{m}$. There is some question about the static stability of an MEB: since the energy of the bubble, defined as the sum of the electrostatic and the surface tension energy, is proportional to $N^{4 / 3}$ [1], clearly the energy of two bubbles with $N / 2$ electrons is lower than that of a single bubble with $N$ electrons. Evidently fissioning is hindered by a formation barrier, since MEBs have been observed [6]. Gravitational fields may flatten very large bubbles and lead to instability [1]. Dexter and Fowler [7] showed that two-electron bubbles are unstable. Salomaa and Williams [2] considered the dynamic stability against fissioning off of single electrons from large bubbles and found stability against this decay mode for bubbles with $N$ greater than 15-20. It is straightforward to show that a positive pressure radially stabilizes a bubble, although angular modes can be unstable. With increasing negative pressure the bubble is first absolutely stabilized and then explodes, as we describe. Salomaa and Williams also considered the dynamic instability due to one of the surface oscillation modes, or ripplons, being soft (zero frequency)
PACS numbers: 67.40.Yv, 47.55.Dz, 73.20. $-\mathrm{r}$

and found that this mode may be stabilized by the anharmonicity in the bubble's radial oscillation that results in a radius larger than $R_{\mathrm{C}}$. In this Letter we study pressure related effects on the frequency of the modes of oscillation, on the equilibrium bubble radius, and on the stability of MEBs; we show the counterintuitive result that positive pressures can destabilize all higher angular modes, while negative pressures have a window of stabilization. We neglect gravity so that the MEBs are spherical.

The frequencies of the modes of oscillation of a charged droplet were first calculated by Rayleigh [8], and in the case of a charged bubble by Plesset and Prosperetti [9]. We first set up a Lagrangian formalism to calculate the spherical ripplon modes. We then consider the effect of pressure on the static and dynamic properties of the bubble. The surface of the bubble is described by a function $R(\theta, \varphi)$ that gives the distance of the surface from the geometrical center of the bubble, in the direction given by the two spherical angles $\theta, \varphi$. This function can be written as $R(\theta, \varphi)=R_{\mathrm{b}}+u(\theta, \varphi)$, where $R_{\mathrm{b}}$ is the angleaveraged radius of the bubble and $u(\theta, \varphi)$ describes the deformation of the surface from a sphere. This deformation can be expanded in a series of spherical harmonic deformations $Y_{\ell m}(\theta, \varphi)$ with amplitude $Q_{\ell m}$,

$$
u(\theta, \varphi)=\sum_{\ell=1}^{\infty} \sum_{m=-\ell}^{\ell} Q_{\ell m} Y_{\ell m}(\theta, \varphi) .
$$

In what follows, we assume that the amplitude of deformation is small, so that for all $\{\ell, m\}, \sqrt{\ell(\ell+1)} Q_{\ell m} / R_{\mathrm{b}} \ll 1$.

The kinetic energy $T$ associated with the motion of the liquid helium surface can be derived from the velocity potential $\psi$. The kinetic energy for incompressible flow of an inviscid fluid is given by

$$
\begin{aligned}
T= & \frac{\rho}{2} \int_{0}^{2 \pi} d \varphi \int_{0}^{\pi} d \theta \psi\left(R_{\mathrm{b}}, \theta, \varphi\right) \\
& \times\left[\left.\mathbf{n} \cdot \nabla \psi(r, \theta, \varphi)\right|_{r=R_{\mathrm{b}}}\right] R_{\mathrm{b}}^{2} \sin \theta,
\end{aligned}
$$

with the density of helium $\rho=145 \mathrm{~kg} / \mathrm{m}^{3}$, and $\mathbf{n}$ is the unit vector normal to the bubble surface. The coefficients 
of expansion in spherical harmonics of the velocity potential are then expressed as a function of the deformation amplitude $Q_{\ell m}$, leading to

$$
T=\frac{\rho R_{\mathrm{b}}^{3}}{2} \sum_{\ell=0}^{\infty} \sum_{m=-\ell}^{\ell} \frac{1}{\ell+1}\left|\dot{Q}_{\ell m}\right|^{2} .
$$

The potential energy of the deformed bubble results from the surface tension, the pressure exerted on the bubble, and the electrostatic forces between the electrons of the spherical 2DEG. The surface tension energy can be written as $\sigma \mathrm{S}$, where $\sigma=3.6 \times 10^{-4} \mathrm{~J} / \mathrm{m}^{2}$ is the surface tension of helium at zero pressure and $\mathrm{S}$ is the area of the deformed surface [8]:

$$
\mathrm{S}=4 \pi R_{\mathrm{b}}^{2}+\frac{1}{2} \sum_{\ell=1}^{\infty} \sum_{m=-\ell}^{\ell}\left(\ell^{2}+\ell+2\right)\left|Q_{\ell m}\right|^{2} .
$$

Deforming the bubble will change its volume and perform $p \mathrm{~V}$ work against the external pressure $p$ of the helium liquid. The volume of the deformed bubble up to second order in the deformation amplitudes is given by [8]

$$
\mathrm{V}=\frac{4 \pi}{3} R_{\mathrm{b}}^{3}+R_{\mathrm{b}} \sum_{\ell=1}^{\infty} \sum_{m=-\ell}^{\ell}\left|Q_{\ell m}\right|^{2} .
$$

The electrostatic energy of the electrons in the spherical 2DEG in the MEB can be derived by taking into consideration that the electrons are strongly confined in the direction perpendicular to the helium surface (the binding energy is of the order of $10 \mathrm{~K}$ ) and anchored to that surface, but free to move in the directions parallel to the helium surface. This leads to the following expression for the Coulomb part $U_{\mathrm{C}}$ of the potential energy [8]:

$$
U_{\mathrm{C}}=\frac{N^{2} e^{2}}{2 \varepsilon R_{\mathrm{b}}}-\frac{N^{2} e^{2}}{8 \pi \varepsilon R_{\mathrm{b}}^{3}} \sum_{\ell=1}^{\infty} \sum_{m=-\ell}^{\ell} \ell\left|Q_{\ell m}\right|^{2},
$$

with $\varepsilon=1.0572$ the dielectric constant of helium. This expression is valid up to second order in the deformation amplitude $Q_{\ell m}$ and does not include exchange or correlation energies of the electron gas.

Collecting the previous expressions [(3)-(6)] for the different energy contributions leads to the following expression for the Lagrangian $\mathcal{L}_{\text {bubble }}=T-\sigma \mathrm{S}-p \mathrm{~V}-U_{\mathrm{C}}$ :

$$
\begin{aligned}
\mathcal{L}_{\text {bubble }}= & \frac{\rho R_{\mathrm{b}}^{3} \dot{R}_{\mathrm{b}}^{2}}{2}-4 \pi \sigma R_{\mathrm{b}}^{2}-\frac{4 \pi}{3} p R_{\mathrm{b}}^{3}-\frac{N^{2} e^{2}}{2 \varepsilon R_{\mathrm{b}}} \\
& +\sum_{\ell=1}^{\infty} \sum_{m=-\ell}^{\ell}\left\{\frac{\rho R_{\mathrm{b}}^{3}}{2} \frac{1}{\ell+1}\left|\dot{Q}_{\ell m}\right|^{2}-\left[\frac{\sigma}{2}\left(\ell^{2}+\ell+2\right)+p R_{\mathrm{b}}-\frac{N^{2} e^{2}}{8 \pi \varepsilon R_{\mathrm{b}}^{3}} \ell\right]\left|Q_{\ell m}\right|^{2}\right\} .
\end{aligned}
$$

The novel part in this Lagrangian as compared to previous treatments $[2,8,9]$ lies in the terms related to the pressure. We return to the harmonic solutions of this Lagrangian after discussing some static properties.

The equilibrium radius of large bubbles, in the absence of deformations, $Q_{\ell m}=0$, can be found by minimizing the potential energy $U=\sigma \mathrm{S}+p \mathrm{~V}+U_{\mathrm{C}}$ as a function of the bubble radius. The potential energy is

$$
\begin{aligned}
U\left(R_{\mathrm{b}}\right)= & \frac{4 \pi}{3} p R_{\mathrm{b}}^{3}+4 \pi \sigma R_{\mathrm{b}}^{2}+\frac{e^{2} N^{2}}{2 \varepsilon\left(R_{\mathrm{b}}-d\right)} \\
& +\frac{N \hbar^{2}}{2 m_{\mathrm{e}} d^{2}}-0.3176 \frac{e^{2} N^{4 / 3}}{\varepsilon\left(R_{\mathrm{b}}^{2} d\right)^{1 / 3}} .
\end{aligned}
$$

For large bubbles, with $N \geqq 10^{3}$, only the first three terms on the right-hand side of (8) play a role - these terms are also present in the Lagrangian (7). The fourth term $\left(m_{\mathrm{e}}\right.$ is the electron mass) is due to Shikin [1] who makes the argument that there is a finite thickness $d \ll R$ of the electron layer that should be taken into account, along with $R_{\mathrm{b}}$, as a variational parameter to minimize the potential energy. The density functional calculations of Shung and Lin [10] show that the exchange energy of the electron gas also plays a relevant role for small bubbles $(N<1000)$ and that for practical purposes it is well approximated by adding an exchange term, the fifth term, on the right-hand side of (8).

The equilibrium radius $R_{\mathrm{eq}}$ of the MEB is found as a function of the number of electrons and the exerted pressure, by minimizing the potential energy $U\left(R_{\mathrm{b}}\right)$ with respect to $d$ and $R_{\mathrm{b}}$. The value of $R_{\mathrm{b}}$ which minimizes the potential energy $U\left(R_{\mathrm{b}}\right)$ is the equilibrium radius $R_{\text {eq }}$ shown in Fig. 1 as a function of pressure for different numbers of electrons. The pressure reduces the equilibrium radius by a significant factor as compared to the zero pressure radius. Most of the change in $R_{\mathrm{eq}}$ occurs at low pressures. The behavior of $R_{\text {eq }}$ as a function of pressure shown in Fig. 1 is similar for numbers of electrons up to $10^{8}$ and larger. The graph extends to negative pressure [11] increasing $R_{\text {eq }}$, since superfluid liquid helium can sustain a substantial negative pressure. For any number of electrons in the bubble, there exists a critical negative pressure making the bubble unstable against runaway expansion. We found that the largest equilibrium radius that can be achieved before the critical underpressure is reached is approximately 1.5 times the equilibrium bubble radius at zero pressure, for any number of electrons from a few hundred to $10^{8}$. When the critical underpressure is reached, the only equilibrium radius is $R_{\mathrm{eq}} \rightarrow \infty$. The critical underpressure appears to be inversely proportional to the number of electrons in the bubble, but this could not be shown explicitly. 


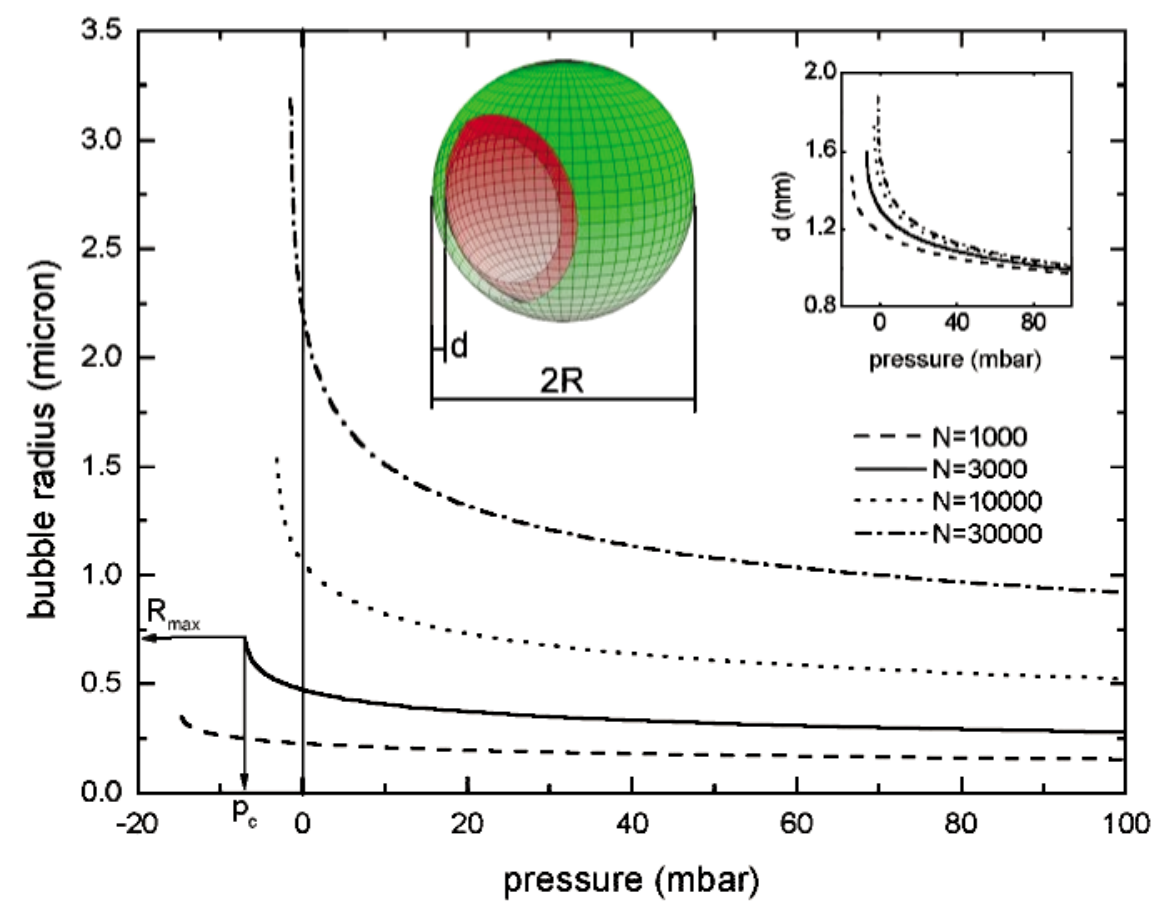

FIG. 1 (color). The equilibrium radius $R$ of a multielectron bubble is shown as a function of pressure for several values of $N$. In the inset, the thickness $d$ of the spherical electron shell in the bubble is shown as a function of pressure for the same numbers of electrons. Both the equilibrium radius and the thickness were obtained by minimizing the potential energy (8). Negative pressure can be applied up to a critical underpressure, expanding the bubble. For $N=3000$ this critical underpressure is indicated with an arrow, as well as the maximum radius of a 3000 electron bubble.

At fixed $R_{\mathrm{b}}$ (the equilibrium radius), the part of the Lagrangian (7) pertaining to the spherical ripplon modes represents a collection of harmonic oscillators in the coordinates $Q_{\ell m}$ with ripplon frequencies

$$
\omega_{\ell}=\sqrt{\frac{\ell+1}{\rho R_{\mathrm{b}}^{3}}\left[\sigma\left(\ell^{2}+\ell+2\right)+2 p R_{\mathrm{b}}-\frac{N^{2} e^{2}}{4 \pi \varepsilon R_{\mathrm{b}}^{3}} \ell\right]} .
$$

These frequencies are independent of the azimuthal index $m$. Figure 2 shows the pressure dependence of the frequency of the spherical ripplon modes $\ell=1, \ldots, 10$. Note first that the $\ell=1,2$ modes have vanishing frequencies at pressures $p \geqslant 0$. This was discussed for zero pressure by Salomaa and Williams [2], who concluded that the $\ell=2$ mode can be dynamically stabilized if the effective radius of the bubble satisfies $R_{\mathrm{b}}>R_{\mathrm{eq}}$ (the $\ell=1$ corresponds to uniform translation). However, the present treatment shows that this argument is no longer valid at increasing pressure. In fact, subsequent modes become unstable, as can be seen from Fig. 2 and expression (9). If the exchange and confinement energy terms in (8) are neglected (a reasonable assumption for bubbles with $N>10^{3}$ ), the equilibrium radius satisfies $2 p R_{\mathrm{eq}}+4 \sigma=e^{2} N^{2} / 4 \pi \varepsilon R_{\mathrm{eq}}^{3}$, so that in this case

$$
R_{\mathrm{b}}=R_{\mathrm{eq}} \Rightarrow \omega_{\ell}=\sqrt{\frac{\ell^{2}-1}{\rho R_{\mathrm{eq}}^{3}}\left[\sigma(\ell-2)-2 p R_{\mathrm{eq}}\right]} .
$$

The pressure at which a mode $\ell>2$ becomes unstable is $p=\sigma(\ell-2) /\left(2 R_{\mathrm{eq}}\right)$. For $N=10^{4}, \sigma / R_{\mathrm{eq}}$ is of the order of 100 mbar. Larger bubbles have even smaller critical pressures. The vanishing of the frequencies of these modes indicates that an instability occurs and that the quadratic approximation for the deformation is no longer valid. Note, however, that a small negative pressure tends to stabilize the $\ell=2$ mode - this is an alternative to Salomaa and Williams' proposal for the stability of the $\ell=2$ mode, based on the assumption that the effective radius $R_{\mathrm{b}}>R_{\text {eq }}$.

In this Letter, we have shown that small negative pressures can stabilize a bubble against dynamic instability, while positive pressures can drive all ripplon modes unstable. Since both negative and positive pressures are easily achievable experimentally, it will be interesting if bubbles can be created in a "stable" configuration that can be visually observed to study these predictions. In the above considerations we have considered the electrons to be a 2DEG, ignoring Wigner crystallization.

We thank S.N. Klimin, V.M. Fomin, and J. Huang for useful comments and discussions. This research has been supported by the Department of Energy, Grant No. DE-FG002-85ER45190, and by the GOA BOF UA 2000, IUAP, the FWO-V Projects No. G.0071.98, No. G.0306.00, No. G.0274.01, No. WOG WO.025.99N (Belgium), and the ESF Programme VORTEX. J.T. is supported financially by the FWO-Flanders. 


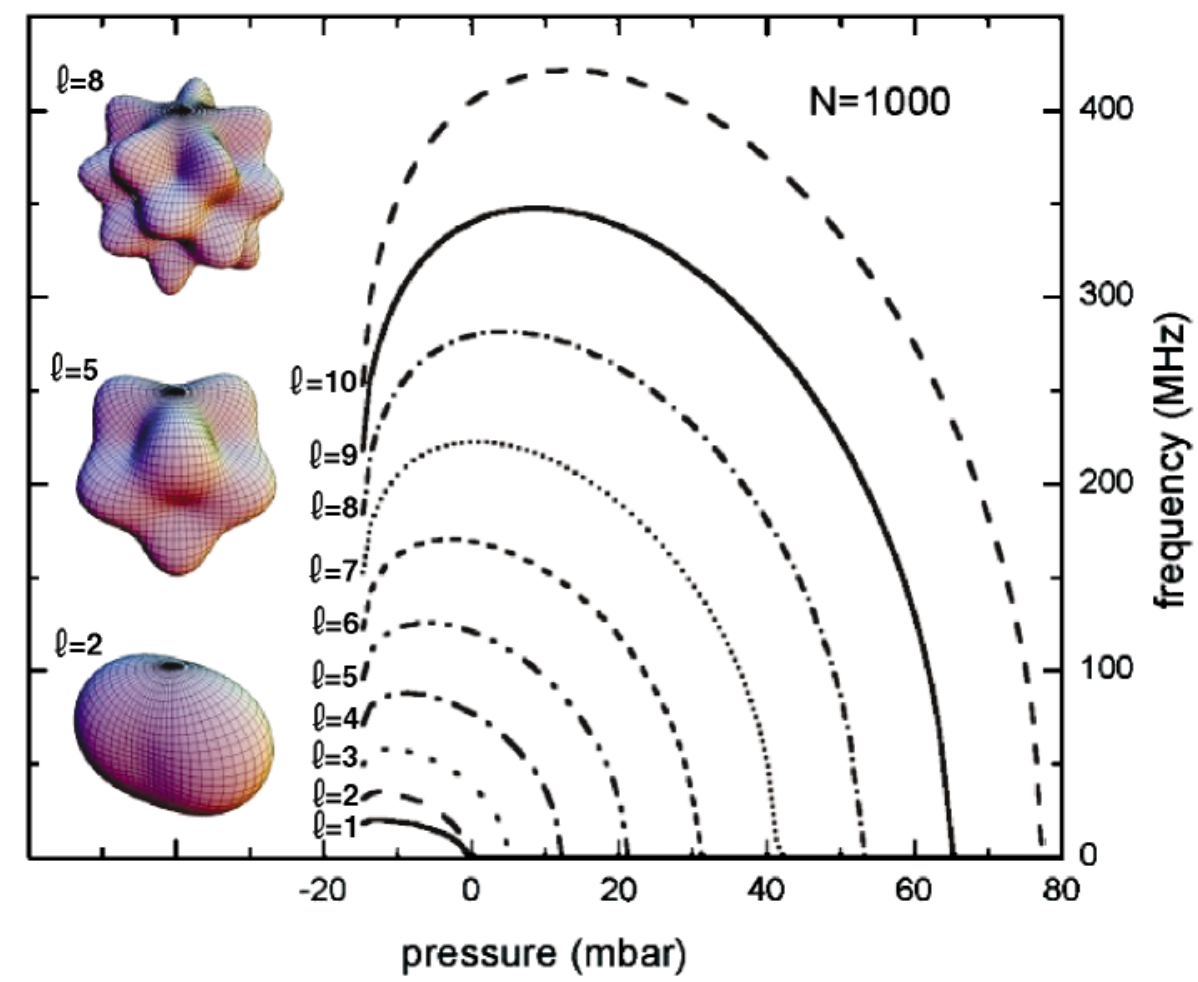

FIG. 2 (color). The frequency of ripplon modes $\ell=1, \ldots, 10$ is shown as a function of external applied pressure (not vapor pressure) for $N=1000$. The leftmost points of the graphs start at the critical underpressure: for a pressure more negative than this value, the bubble is unstable against isotropic expansion. As the pressure is increased, more and more modes obtain a vanishing frequency. On the left the deformations (exaggerated) are shown for a few of the modes.

*Also at Technische Universiteit Eindhoven, Postbus 513, 5600MB Eindhoven, The Netherlands.

[1] V. B. Shikin, JETP Lett. 27, 39 (1978).

[2] M. M. Salomaa and G. A. Williams, Phys. Rev. Lett. 47, 1730 (1981).

[3] M. W. Cole, Rev. Mod. Phys. 46, 451 (1974).

[4] I. F. Silvera, Bull. Am. Phys. Soc. 46, 1016 (2001).

[5] C. C. Grimes and G. Adams, Phys. Rev. B 41, 6366 (1990); J. Classen, C.-K. Su, M. Mohazzab, and H. J. Maris, Phys. Rev. B 57, 3000 (1998).

[6] A. P. Volodin, M. S. Khaikin, and V.S. Edel'man, JETP Lett. 26, 543 (1977); U. Albrecht and P. Leiderer, Europhys. Lett. 3, 705 (1987).
[7] D. L. Dexter and W. B. Fowler, Phys. Rev. 183, 307 (1969).

[8] Lord Rayleigh, Proc. R. Soc. London 29, 71 (1879); Philos. Mag. 14, 184 (1882).

[9] M. S. Plesset and A. Prosperetti, Annu. Rev. Fluid Mech. 9, 145 (1977).

[10] K. W. K. Shung and F. L. Lin, Phys. Rev. B 45, 7491 (1992).

[11] I. F. Silvera, J. D. Gillaspy, and J. G. Brisson, Spin Polarized Quantum Systems, edited by S. Stringari (World Scientific, Singapore, 1989), p. 89; H. J. Maris and Q. Xiong, Phys. Rev. Lett. 63, 1078 (1989). 